\title{
Féeries
}

Études sur le conte merveilleux, XVII ${ }^{\mathrm{e}} \mathrm{XIX} \mathrm{X}^{\mathrm{e}}$ siècle

1 | 2004

Le Recueil

\section{La mise en recueil des Mille et un jours}

\section{Christelle Bahier-Porte}

\section{OpenEdition}

\section{Journals}

Édition électronique

URL : http://journals.openedition.org/feeries/72

DOI : $10.4000 /$ feeries.72

ISSN : 1957-7753

Éditeur

UGA Éditions/Université Grenoble Alpes

Édition imprimée

Date de publication : 1 février 2004

Pagination : 93-106

ISSN : 1766-2842

Référence électronique

Christelle Bahier-Porte, «La mise en recueil des Mille et un jours ». Féeries [En ligne], 1 | 2004, mis en ligne le 29 mars 2007, consulté le 08 septembre 2020. URL : http://journals.openedition.org/feeries/72 ; DOI : https://doi.org/10.4000/feeries.72

Ce document a été généré automatiquement le 8 septembre 2020

(c) Féeries 


\title{
La mise en recueil des Mille et un jours
}

\author{
Christelle Bahier-Porte
}

1 LES MILLE ET UN JOURS, CONTES PERSANS paraissent en cinq volumes entre 1710 et 1712. L'éminent orientaliste François Pétis de La Croix, avec la collaboration probable de l'écrivain Alain-René Lesage ${ }^{1}$, reprend explicitement la structure des Mille et Une Nuits, contes arabes, publiés depuis 1704 par Antoine Galland. Le récit-cadre met ainsi en scène une conteuse qui captive ses auditrices par ses histoires tous les matins à l'heure du bain. La structure est donc inversée par rapport aux Mille et Une Nuits: la nourrice Sutlumemé propose des contes de jour et s'adresse à une jeune femme, Farrukhnaz qui, après un songe, refuse de croire à la fidélité des hommes et par conséquent refuse de se marier. L'enjeu des contes est certes bien moindre que dans Les Mille et Une Nuits, il n'est pas question de vie ou de mort ni même de succession politique puisque le roi de Cachemire a un fils. Il n'empêche que d'un strict point de vue structurel, Pétis de La Croix reprend le principe du recueil de contes arabes. Il reproche en outre à Antoine Galland le manque de cohérence des contes arabes et prétend proposer une unité pour son propre recueil. Ce lien entre les contes sera d'ordre moral : il s'agit de prouver, par des histoires, qu'il y a des hommes fidèles et ainsi de conduire la jeune princesse au mariage :

Les lecteurs qui, dans les contes arabes, ont trouvé mauvais qu'on n'ait pas donné à Schéhérazade une intention de persuader par ses fables à Schariar qu'il $\mathrm{y}$ a des femmes fidèles [...] ceux, dis-je, qui ont fait cette critique ne feront pas le même reproche à dervis Moclès. Sutlumemé se propose de combattre la prévention de la princesse et va toujours à sa fin. Dans tous ses contes, il y a des époux ou des amants fidèles. On voit qu'elle s'applique à guérir Farrukhnaz de son erreur ${ }^{2}$.

2 Si cette perspective est globalement respectée, elle est cependant parfois mise à mal et ce sont ces accidents dans l'apparente cohérence du recueil de contes persans qui vont nous intéresser.

3 La notion de " recueil » se révèle en tout état de cause particulièrement opératoire pour Les Mille et Un Jours. Pétis de La Croix, comme Galland avant lui, s'attelle à une «collecte» de contes de différentes origines auxquels il choisit de donner une 
cohérence. Si notre propos n'est pas ici d'étudier l'exploitation des sources, il sera cependant utile de rappeler comment l'orientaliste construit son recueil à partir d'un matériau d'une part authentique et d'autre part fort disparate. C'est néanmoins le choix de cette " cohérence » postulée par le rédacteur de la préface, et au fondement de notre perception du «recueil», qu'il nous appartiendra ici d'interroger afin d'en mettre en lumière tous les aspects. Loin d'être un faux recueil ou une entreprise commerciale qui profiterait du succès des contes arabes, Les Mille et Un Jours se fondent peut-être sur une logique secrète, moins apparente que la nourrice voudrait le laisser croire, sensible dans l'analyse des failles d'un recueil trop parfaitement ordonné. Sous l'ordre moral affirmé se cacherait une logique du désir qui permettrait de mettre en question le sens du recueil: la fidélité masculine serait moins la clé du recueil que l'insatiable quête du bonheur intrinsèque à la nature humaine. La mise en recueil de contes à l'origine disparates serait alors elle-même emblématique de cette quête: refuser la satisfaction pour toujours réactiver le désir, d'écouter et de lire.

Depuis le XIX ${ }^{e}$ siècle, on sait que le recueil repose sur une supercherie. Pétis de La Croix affirme dans sa préface qu'il tient les contes d'un certain derviche nommé Moclès qu'il aurait rencontré lors de son voyage à Ispahan. Si l'orientaliste a bien rencontré cet homme ${ }^{3}$, il n'y a aucune trace d'un échange de contes, et on n'a jamais pu retrouver le fameux recueil Hezaryek-Rouz invoqué dans la préface. Les orientalistes, Joseph Hammer, Spencer Smith, Loiseleur-Deslongchamp ou encore, plus récemment, Paul Sebag ont tour à tour montré que le recueil de Pétis de La Croix repose sur un assemblage savant de contes, pour la plupart empruntés au recueil turc Al-farage bada al-schidda également mentionné dans la préface. À quoi bon inventer cet échange avec le dervis Moclès ${ }^{4}$ ? Dans la perspective qui est la nôtre, on peut dire que l'orientaliste place ainsi son recueil sous la tutelle d'une figure unique et inscrit en quelque sorte l'origine du recueil dans un échange oral, d'homme à homme, comme la nourrice va être une nouvelle figure de la conteuse orale. Le dervis Moclès serait une première preuve, en amont, du souci de cohérence du recueil.

Quoi qu'il en soit, pour composer son recueil de contes persans, Pétis de La Croix choisit et réécrit seize des quarante-deux contes du recueil turc, qu'il a tout simplement trouvé dans la bibliothèque royale. Quelques histoires des Mille et Un Jours sont la transformation d'un seul conte-source. Par exemple, le conte $\mathrm{n}^{\circ} 5$ du manuscrit turc intitulé «Farrukschad, Farrukhruz et Farrukhnaz » est à l'origine du récit-cadre. Le conte $n^{\circ} 25$ intitulé «Le prince Calaf et la fille de l'Empereur de la Chine » devient «l'Histoire du prince Calaf et de la princesse de la Chine »; le conte n ${ }^{\circ} 26$ intitulé « Le jardin de Iram, Badi al Jamal et Sayf el Muluk» fonde l'Histoire de Seyf-el-Mulouk, même si Pétis de La Croix en modifie le dénouement pour faire correspondre le conte à l'ensemble thématique formé par la quête d'un homme heureux du roi Bedreddin-Lolo ${ }^{5}$. Le conte turc $\mathrm{n}^{\circ} 12$ « La femme de l'orfèvre, le faqih, le cadi, le muhtasib, le préfet et le gouverneur" donne la plaisante "Histoire de la belle Arouya». Le plus souvent cependant les contes " persans » sont issus d'une recomposition de différentes sources. Ainsi l'« Histoire d'Aboulcassem Basri» s'inspire de trois contes du Farage bada alschidda: le conte $\mathrm{n}^{\circ} 1$ pour la générosité fabuleuse du marchand, le conte $\mathrm{n}^{\circ} 16$ pour le trésor inépuisable, le conte $\mathrm{n}^{\circ} 2$ pour le récit des amours d'Aboulcassem et Dardané ; s'y ajoute probablement un conte de l'Histoire des quarante vizirs pour l'épisode de la dame assassinée. De la même façon, l'« Histoire d'Atalmuk surnommé le vizir triste » est le résultat du croisement de trois contes turcs : le conte $n^{\circ} 17$ pour les aventures du vizir sous le nom d'Hassan, le conte $n^{\circ} 11$ pour le récit central et le conte $n^{\circ} 10$ pour le 
dénouement et notamment le pardon du roi. Pétis de La Croix peut aussi utiliser le même conte turc pour plusieurs contes persans: ainsi le conte $\mathrm{n}^{\circ} 11$ est mis à contribution pour la quête de Bedreddin-lolo qui sert de deuxième récit-cadre - nous y reviendrons -, pour le conte d'Hormoz et pour celui d'Atalmuk. Le conte $n^{\circ} 2$ enrichit le conte d'Aboulcasssem et celui de Fadlallah. Enfin, outre ces contes turcs, Pétis de La Croix exploite d'autres sources dont certaines sont encore mystérieuses. Les orientalistes ont ainsi reconnu des épisodes issus de l'Histoire des quarante vizirs dans l'histoire d'Aboulcassem, du vizir Caverscha, de Couloufe et Dilara ou encore de Fadlallah. Le récit inséré de la vie du philosophe Avicène dans l'histoire d'Hormoz s'inspire du Roman d'Avicène (xvI siècle). En revanche, l'origine de l'« Histoire des deux frères génies Adis et Dahy ", probablement indienne, reste à ce jour inconnue de même que celle de la fin de l'histoire du jeune roi de Tibet $^{6}$.

Ce ne sont ici que quelques exemples pour prendre la mesure du travail de collecte, livresque, effectué par l'orientaliste pour construire son recueil. Un conte persan naît ainsi de l'« amplification », au sens rhétorique du terme, d'un conte turc - Paul Sebag en donne un exemple intéressant en publiant pour la première fois une traduction littérale du manuscrit turc à la suite du conte « traduit » par Pétis de La Croix ${ }^{7}-$, mais le plus souvent le conte persan est issu de la recomposition de plusieurs contes. Ce travail d'assemblage et de réécriture pour aboutir à une cohérence interne des contes à partir de leurs sources reste pour une bonne part à apprécier, notamment à partir des traductions de manuscrits par les jeunes de langue du XvIII ${ }^{e}$ siècle disponibles à la bibliothèque nationale ou encore, avec les compétences requises, à partir des manuscrits orientaux ${ }^{8}$. Nous nous intéresserons pour notre part au travail de composition du recueil, c'est-à-dire aux liens ménagés entre les contes. Comment Pétis a-t-il transformé la collecte en recueil ?

7 On peut a priori solliciter deux sortes d'analyses. La première serait d'ordre narratologique, la seconde, appelée par la déclaration liminaire, serait d'ordre thématique puisque Pétis de La Croix postule la vocation morale de son recueil. On peut tout d'abord se demander si l'ordre des contes, leur progression, leur «disposition » sont porteurs de sens.

8 Le principe organisateur essentiel qui fonde notre perception de la collecte de contes comme «recueil» est sans nul doute l'encadrement. Cet encadrement est d'abord visible par la présence d'une narratrice-conteuse. Chaque histoire est introduite et conclue par la nourrice. Elle demande à la princesse l'autorisation de prendre la parole puis commence son histoire. On lit par exemple : « je vous le ferai voir par l'histoire de Couloufe et de la belle Dilara, si vous me permettez de vous la raconter. Je le veux bien, reprit la princesse. [...] Alors la nourrice commença de cette manière ${ }^{9}$ ». La fin du conte est en outre soulignée par les réactions des auditrices qui rappellent, en mineur, les débats qui animaient la fin des contes du Décaméron ou de l'Heptaméron:

Sutlumemé finit en cet endroit l'histoire d'Aboulcassem Basri. Toutes les femmes de la princesse de Cachemire lui donnèrent de grands applaudissements: les unes louèrent la magnificence et la générosité de ce jeune homme de Basra; les autres prétendaient que le calife Haroun-Arraschid n'était pas moins généreux que lui ; d'autres enfin, ne s'attachant qu'à la constance, disaient qu'Aboulcassem avait été un amant très fidèle. Alors Farrukhnaz prenant la parole dit: «je ne suis pas de votre sentiment $[\ldots] »^{10}$.

9 On trouve encore à la fin de l'histoire de Bedreddin-Lolo: «la princesse, selon sa coutume, ne manqua pas de trouver à redire à leur fidélité. Cela ne rebuta point la 
nourrice qui demanda la permission de conter de nouvelles histoires. Elle l'obtint et le jour suivant, elle reprit la parole de cette manière » (p. 407). Pétis de La Croix atténue cependant les transitions entre les jours - les récits devant s'achever à la prière de midi -, afin d'éviter l'ennui du lecteur. Il s'en explique à la fin du premier jour : «c'est de cette manière que le dervis Moclès a fait la division de ces contes. On a suivi cet ordre, mais on a retranché tout ce qui dans l'original est devant et après la narration essentielle parce que cela ne sert qu'à la faire languir et qu'à ennuyer le lecteur qui, par ce retranchement, lira les contes sans s'apercevoir qu'ils sont interrompus ${ }^{11} »($ p. 43). Cette affirmation est aussi une manière, par prétérition, de consacrer la filiation avec Galland, de la nuit au jour, et de postuler un « ordre " préexistant à la mise en recueil du traducteur français. Elle confirme donc le souci de cohérence de ce traducteur.

Le récit-cadre du recueil, c'est-à-dire l'histoire de la princesse de Cachemire, de son aversion pour le mariage à sa conversion grâce aux manœuvres du prince de Perse et de son confident, a été recomposé par Pétis de La Croix afin de placer en son centre un personnage féminin ${ }^{12}$. L'orientaliste invente alors un second personnage féminin: la nourrice Sutlumemé qui prétend se charger de la conversion de la princesse grâce à ses histoires: «je sais une infinité d'histoires curieuses dont le récit, en divertissant la princesse, lui ôtera la mauvaise opinion qu'elle a des hommes » (p. 41). Les contes sont donc destinés à des femmes dans l'intimité du bain. Le dessein de la conteuse est privé : faire le bonheur de la jeune princesse en lui faisant accepter le mariage.

11 Cependant, cet encadrement est dédoublé par un second récit-cadre, à l'intérieur même des histoires de Sutlumemé. Il ne s'agit pas d'introduire un narrateur second mais un auditoire second. En effet, le roi Bedreddin-Lolo refuse de croire à l'affirmation de son vizir : il n'y a pas un seul homme parfaitement heureux sur la terre. Pour prouver le contraire, il fait d'abord défiler devant lui tous ses officiers, tous ses courtisans et enfin son peuple tout entier. Chacun va lui raconter son histoire, lui révéler le « fond de son âme $^{13}$ » et finalement avouer son malheur ${ }^{14}$. Le roi veut alors partir sur les routes avec son vizir et son favori afin de trouver un homme heureux. Cette quête le mènera jusqu'à Hormoz, le "roi sans chagrin", puis Aboulfaouaris, "le grand voyageur ". L'histoire de Bedreddin-Lolo encadre les histoires d'Atalmuk, de Seyf-el-Mulouk, de Malek, d'Hormoz, de la belle Arouya et d'Aboulfaouaris. Il n'est pas inapproprié de parler de second récit-cadre car ces contes occupent presque le même volume que les histoires autonomes de la nourrice ${ }^{15}$ et on n'observe aucune intervention de l'auditoire de la nourrice - la princesse et ses suivantes -, pendant tout le cycle de Bedreddin-Lolo. Le dessein est donc bien différent. Il est certes toujours question de bonheur, et ce bonheur est dans la plupart des récits lié à la satisfaction amoureuse, mais la quête du roi pose aussi le problème du possible bonheur du gouvernant, d'une association possible entre bonheur et pouvoir, privé et public et on touche là des questionnements essentiels du siècle. Il n'est pas sûr que la jeune Farrukhnaz soit intéressée par ce problème et c'est pourquoi la nourrice prend soin de lier la problématique à l'amour. Par exemple, Seyf-el-Mulouk, amoureux d'une princesse morte depuis des siècles est, en dépit de cet " amour singulier » présenté comme un exemple de fidélité. Il conclut en effet son histoire en ces termes : «j'ai beau me représenter à tous moments que c'est une extravagance à moi d'en être amoureux comme d'une dame qui serait en vie, il m'est impossible de triompher de son image : elle règne toujours dans mon cœur » ( $p$. 267). Le thème est néanmoins présent, ainsi Bedreddin reconnaît d'emblée qu'il n'est pas parfaitement heureux du seul fait de sa condition de roi: "Pour moi, s'écria 
Bedreddin, je ne puis l'être [parfaitement content] ; j'ai des ennemis sur les bras ; je suis chargé du poids d'un empire : mille soins partagent mes esprits et troublent le repos de ma vie ; mais je suis persuadé qu'il y a dans le monde une infinité de particuliers dont les jours heureux coulent dans des plaisirs qui ne sont mêlés d'aucune amertume » (p. 214). De la même façon, il refuse de croire que le roi Hormoz puisse être réellement heureux: «je doute comme vous qu'un homme chargé du poids d'un état soit sans chagrin » (p. 288).

Cette rencontre de la quête masculine (Bedreddin) et de la quête féminine (Farrukhnaz), du pouvoir et de l'amour, du public et du privé est peut-être à l'origine du changement d'orientation du recueil à ce moment précis. Roger Laufer l'avait déjà remarqué et il qualifie le cycle de Bedreddin-Lolo de "contre-thème » et ainsi le met directement en rapport avec le projet de la nourrice : «Lesage ${ }^{16}$ relance la série des contes par le contre-thème du roi Bedreddin-Lolo. [...] Le thème et le contre-thème présentent donc avec enjouement la double question d'amour : qui aime avec plus de constance des femmes ou des hommes $?^{17}$ ». Roger Laufer en reste donc à la thématique amoureuse qui, selon lui, engage toute la structure des contes et du recueil selon un schéma de type proppien: situation initiale/obstacles/résolution ${ }^{18}$. Pourtant, si on accepte l'idée d'un « second recueil » avec l'histoire-cadre de Bedreddin-Lolo, cet écart par rapport au projet initial prend tout son sens et invite à reconsidérer l'unité postulée du recueil : est-elle seulement fondée sur la démonstration de la fidélité des hommes ? Si on la remet en cause, on comprend mieux la focalisation de la deuxième partie du premier récit-cadre sur les aventures du prince de Perse. À l'attente féminine, répond l'action masculine : à la résignation succède l'action. En effet, après le récit de la quête de Bedreddin-Lolo, la quête du prince de Perse prend un nouveau sens. Certes, il cherche la belle princesse qu'il a vue en rêve, mais il cherche surtout la satisfaction de son désir dont le rêve serait, si l'on peut dire, la " matérialisation ", un désir qu'il refuse de croire une chimère, comme Bedreddin refuse de croire qu'il n'existe pas d'homme heureux. C'est Symorgue, le confident, qui engage son maitre à partir sur les routes chercher cette belle princesse, même s'il avoue que c'est pour mieux le divertir de son « objet chimérique» (p. 473). Pourtant, il se laisse lui-même prendre au désir de son maître et déclare à la magicienne Ghulnaze: "Que deviendra mon cher prince ? Comment pourra-t-il obtenir l'accomplissement de ses désirs $?^{19} »($ p. 476$)$. L'unité du recueil, le fil qui relierait tous les contes, serait moins la fidélité exemplaire que la recherche du bonheur, plus précisément la notion même de quête et d'espoir, que celui-ci soit déçu (Bedreddin) ou comblé (le prince de Perse). Le recueil nous parlerait tout simplement du désir, moteur des actions de la nature humaine. Bedreddin affirmait d'ailleurs sa confiance dans la nature humaine pour justifier sa quête : «je ne puis croire ce que vous me dites [...] j'ai meilleure opinion de la nature humaine, et je suis persuadé qu'il y a des personnes dont le repos n'est troublé par aucun chagrin » (p. 267). Les songes qui encadrent le recueil : celui de Farrukhnaz et celui du prince de Perse, se rencontrent au dénouement : « [Symorgue] conduisit son maître à Farrukhnaz qui reconnut dans le prince les traits qu'elle avait vus en songe : comme de son côté, Farrukhschad connut d'abord, en la regardant, que c'était la princesse dont il conservait si chèrement l'image ${ }^{20} »($ p. 480). L'objectif du recueil serait finalement de faire coïncider deux désirs.

13 L'agencement des contes en un récit-cadre premier qui accueille un récit-cadre second nous invite alors à examiner la cohérence thématique du recueil. Pétis de La Croix l'affirme dans sa préface: il s'agit de prouver qu'il y a des hommes fidèles. C'est le 
programme de Sutlumemé, plusieurs fois réitéré. Ainsi, la princesse de la Chine, qui elle aussi refuse de se marier, doit " vaincre cette aversion si contraire à la nature » (p. 206). Les histoires malheureuses sont particulièrement exemplaires pour la nourrice. Fadlallah dont l'épouse s'est laissée mourir après avoir appris qu'elle avait trompé son époux dont un derviche avait emprunté les traits achève son histoire sur ces mots: "Zemroude est toujours présente à ma pensée et je prends plaisir à m'en ressouvenir " (p. 170). Dans le cycle de Bedreddin-Lolo, Atalmuk déclare : « l'enlèvement de Zélica est toujours présent à ma pensée et me rend insensible à la joie» (p. 247). Les contes seraient donc des "miroirs de princesses ${ }^{21}$ » enseignant les valeurs morales: la générosité, la clémence, la constance tout en défendant la réalité de la fidélité masculine. L'analyse de Marie-Louise Dufresnoy va dans ce sens puisqu'elle dote chaque histoire d'un sous-titre moral, à l'instar des exempla occidentaux. Ainsi l'histoire d'Aboulcassem pourrait s'intituler «La générosité récompensée », celle de Calaf: «La piété filiale récompensée »; la belle Arouya qui part solliciter les débiteurs de son époux trop libéral serait un exemplum de fidélité conjugale ${ }^{22}$. C'est en outre un véritable miroir que la nourrice tend à la jeune princesse en faisant le portrait de Tourandocte, princesse de la Chine ou de Rézia, fille du sultan de Carizme. On apprend en effet au début du récit-cadre que Farrukhnaz «inspirait de l'amour à tous les hommes qui osaient la regarder ; mais cet amour leur devenait funeste, car la plupart en perdaient la raison ou tombaient dans une langueur qui les consumait insensiblement » (p. 39). La beauté de la princesse Rézia rend également les hommes fous, à tel point que son père a fait construire des tours pour les accueillir ${ }^{23}$. Lorsque Farrukhnaz sort sans voile pour aller à la chasse, les prétendants meurent d'amour pour elle. De la même façon, le père de Rézia a fait publier un édit pour empêcher les hommes de regarder sa fille quand elle joue au mail sans voile : « De la part du sultan, j'avertis le peuple que la princesse Rézia joue au mail. Si quelqu'un a l'imprudence de la regarder, je déclare qu'il ne pourra s'imputer qu'à lui-même le mal qui lui en arrivera » (p. 299). La princesse de Cachemire obtient le serment que son père ne la mariera pas contre son gré, le père de Tourandocte doit quant à lui publier un édit affirmant que sa fille n'épousera que celui qui sera capable de résoudre trois énigmes ${ }^{24}$. Tourandocte et Rézia se laissent pourtant séduire, la première par le prince Calaf la seconde par le roi Hormoz et, en vertu du pouvoir performatif que la nourrice accorde au conte, Farrukhnaz est invitée à imiter ses doubles. Pourtant Sutlumemé échoue puisque la princesse n'est pas convaincue après mille et un jours de contes. Il faudra une manigance masculine et une peinture représentant l'inverse du songe funeste de la jeune fille pour la convertir à l'amour. Il faudrait croire alors que l'image a plus de pouvoir que la parole ou que l'auteur du recueil se moque discrètement du dénouement conventionnel des contes, comme du prétendu « pouvoir des fables ${ }^{25}$ ».

Le titre original du recueil, non Hezaryek-Rouz, "Les Mille et Un Jours », mais Al-farage bada al-schidda, "La joie après l'affliction » donne alors un second fil pour relier les contes. L'histoire du vizir Caverscha insérée dans l'histoire de Fadlallah n'a aucun rapport avec la problématique amoureuse et apparaît comme une parabole de la conception de la vie humaine exposée dans le recueil, liée à une certaine forme de fatalisme. Caverscha, vizir du roi d'Hircanie, voit un jour sa bague flotter dans l'eau du bain. Il en déduit qu'il va se faire arrêter ; et c'est en effet ce qui arrive à la suite d'un faux témoignage. Après plusieurs années de prison, on lui apporte son plat favori qu'il avait toujours réclamé en vain; deux gros rats tombent dedans. Le vizir en déduit qu'il va être libéré: «les rats qui sont tombés dedans m'ont fait connaître que j'étais 
parvenu aux bornes prescrites à ma mauvaise fortune et que ma douleur extrême serait bientôt suivie d'une parfaite joie" (p. 89). Cette alternance d'affliction et de joie caractérise bon nombre de contes ${ }^{26}$. Le début de l'histoire d'Atalmuk en est un exemple. Le jeune homme se pend à une branche pour en finir avec sa vie de débauche; la branche casse et découvre un trésor de pierres précieuses : «j'ôtai vite de mon cou le nœud coulant et passai du désespoir à la joie la plus vive » (p. 216). Ce type d'expression est récurrent : il peut souligner une péripétie, par exemple dans l'histoire de Seyf-elMulouk : « une joie parfaite succéda bientôt à la terreur que les nègres nous avaient inspirée ; et riant des choses mêmes qui nous avaient épouvantés, nous nous mîmes à plaisanter sur les bonnes fortunes ${ }^{27}$ que nous avions dédaignées » (p. 258), ou consacrer un dénouement, par exemple lorsque le roi de Moussel rend Zeïneb à son ami : « Hâtez vous de joindre Nasiraddolé et de faire succéder dans son cœur la joie la plus vive à l'affliction dont il est saisi » (p. 442). Les deux fils thématiques, l'amour fidèle et la joie après l'affliction, se croisent. Dans la logique de la nourrice, la joie sera le mariage après l'affliction des épreuves endurées pour l'obtenir. Les contes mettent autant en scène la fidélité des amants que leur opiniâtreté à trouver le bonheur, un bonheur selon leur désir. Or, ce que nous apprend le cycle de Bedreddin-Lolo c'est qu'il n'y a pas de bonheur possible sur terre, il faut se contenter de son sort ou se fabriquer un bonheur par provision, au sens cartésien du terme : « les personnes les plus heureuses sont celles dont les peines sont les plus supportables. Demeurons désormais ici tranquilles. Si nous ne sommes pas tous trois pleinement satisfaits, songeons qu'il y en a de plus malheureux » (p. 407). Telle est la conclusion, quelque peu décevante peutêtre, de la longue quête du roi Bedreddin-Lolo.

Il me semble que ces deux éléments : la présence d'un double récit-cadre, le croisement de deux fils thématiques met en question le sens du recueil. D'un côté la nourrice veut nous faire croire à une possible satisfaction: l'amour fidèle existe, mes histoires parfaitement structurées le miment. L'auditeur est ainsi satisfait, il détient toutes les réponses à la fin de l'histoire. Les effets de symétrie sont en effet particulièrement appuyés afin d'insister sur la résolution de la situation initiale. Deux exemples seront suffisants. Au début de l'histoire d'Aboulcassem, c'est Zobéide qui pousse son époux, le calife Haroun-Arraschid, à se rendre à Basra pour vérifier la légendaire générosité du marchand. À la fin du conte, Aboulcassem est convié chez la sultane et c'est grâce à elle qu'il retrouve Dardané ; c'est encore Zobéide qui fait écrire « en lettres d'or » l'histoire des deux amants. L'histoire de Repsima présente une autre forme de symétrie. À la fin du conte, l'héroïne est devenue la reine et l'oracle d'une île gouvernée par des femmes. Elle voit défiler devant elle tous ses anciens ennemis, devenus infirmes. Après avoir entendu leurs aveux, qui sont autant de rappels du conte qu'on vient de lire, elle leur pardonne et les guérit. La symétrie sert ici une intention apologétique, l'histoire s'achève dans une sorte de béatitude générale et de paix, intérieure et politique. Cette reine, sainte et clémente, est bien sûr un nouvel exemple pour Farrukhnaz. La nourrice cherche ainsi la satisfaction de son auditoire, thématisée à l'intérieur du récit par la satisfaction des héros ou leur résignation en ce qui concerne le cycle de BedreddinLolo. D'un autre côté, l'ordonnateur du recueil semble plutôt mettre en avant l'insatisfaction en croisant subtilement les deux fils thématiques : amour et bonheur. Si on met à part le récit-cadre, le recueil progresse en trois temps. On trouve d'abord les contes relevant du premier thème: la fidélité des amants est récompensée par un mariage heureux. Viennent ensuite les contes plus proches du second thème - le « contre-thème » de Roger Laufer -, encadrés par l'histoire de Bedreddin-Lolo : malgré 
leur fidélité, les amants ne peuvent être heureux, même s'ils restent constants. Suivent alors deux contes souriants racontés au Calife Haroun-Arraschid : l'histoire des frères génies et l'histoire de Nasiraddolé, puis le recueil s'achève sur l'histoire édifiante de Repsima. On peut alors discerner un trajet : la satisfaction sensuelle et le mariage sont les premières récompenses des amants. Mais il faut aller au-delà : le malheur seul fait éprouver la valeur de la constance. Les deux histoires souriantes rétablissent alors la joie après l'affliction avant la félicité divine atteinte par Repsima. C'est pourquoi la mélancolie qui émane du cycle de Bedreddin-Lolo, recueil dans le recueil, n'est pas un contresens mais bien une réflexion, nous n'osons pas dire philosophique mais il suffirait de peu pour qu'elle le devienne, sur la nature « désirante » de l'homme : « telle est la condition des enfants d'Adam: notre cœur ne saurait jouir d'une entière satisfaction» (p. 214), « les hommes peuvent-ils jouir d'un sort agréable?» (p. 246), « rien ne peut contenter le cœur humain. À peine a-t-il obtenu l'accomplissement d'un désir qu'il avait formé qu'il sent naître un autre désir qui trouble son repos » (p. 287). Telles sont bien quelques-unes des réflexions qui émaillent les récits du cycle de Bedreddin-Lolo.

16 Ainsi, si le conte tel que le conçoit la nourrice Sutlumemé doit procurer la satisfaction, le recueil la met en question. Finalement, l'exigeante Farrukhnaz qui trouve à redire à toutes les histoires de fidélité de sa nourrice serait une très bonne lectrice de contes, plus sagace que ses suivantes trop vite conquises. Elle est celle qui ne s'en laisse pas conter : « ma chère Sutlumemé, vous avez beau peindre les hommes avec les plus belles couleurs, leurs défauts percent toujours au travers de vos peintures» (p. 214). L'insatisfaction était, au contraire de la nourrice, l'arme principale de Schéhérazade : il lui fallait tenir le désir du sultan éveillé pour sauver sa propre vie et le destin de sa nation. Plus largement, tout « recueil » repose sur l'insatisfaction : si le conte apporte une réponse, lève les obstacles et donne même une leçon de morale à qui veut bien l'entendre, la succession des contes met en question cette clôture apparente. C'est peut-être aussi la fonction des faux dénouements que de participer à cette remise en cause : ce n'est pas seulement la morale qui est mise malicieusement en question par les soupçons qui pèsent sur Zelica ou sur Canzade mais aussi la fonction du conte, qui n'est pas parvenu à la satisfaction précisément. Aboulfaouaris finit son histoire sur son retour à Basra et ses retrouvailles avec son épouse Canzade. L'ordonnateur du recueil choisit alors d'attendre la « fin de l'histoire de Bedreddin-Lolo et de son vizir » pour ajouter une " circonstance » : Canzade aime encore le jeune homme qu'elle avait épousé lorsqu'elle croyait son premier époux mort. La satisfaction du "grand voyageur" devenu conteur n'était donc qu'apparente ${ }^{28}$. La question posée par un recueil est bien de savoir si la multiplication des récits conduit au ressassement, et la logique de la nourrice y conduit inévitablement, ou à la multiplication des désirs: une histoire correspond à un désir satisfait mais la suivante réactive ce désir d'écouter, d'être surpris ou charmé. Lorsque la princesse des Naïmans commence son récit par une énigme, elle ne fait qu'attiser le désir de son auditeur et en bonne conteuse sait ménager ses effets : « je suis fille et femme de roi et cependant je ne suis point ce que je dis. Je suis princesse et ne suis point ce que je suis » (p. 83). Deux conceptions du conte se rencontrent alors dans ce recueil. Le conte aurait une finalité, une légitimité extérieure à lui-même : convaincre ou amuser, instruire et plaire. On retrouve alors la logique du conte "aristocratique » de la fin du dix-septième siècle ${ }^{29}$ et d'ailleurs Pétis de La Croix aurait composé son ouvrage pour Marie-Adélaïde de Savoie, duchesse de Bourgogne. Dans une autre perspective, le conte aurait une légitimité disons 
« interne »: on ne raconte pas "pour » quelque chose, mais on raconte ; parce que tout homme est doté d'une "fonction fabulatrice » dirait Lévi-Strauss mais aussi parce que le conte apporte la joie après l'affliction dirait Beddredin-Lolo. Les Mille et Un Jours thématisent ces deux conceptions du conte grâce à leur double récit-cadre et au récit d'une double quête : l'amour fidèle et le bonheur dont l'issue est tout aussi incertaine. Indéniablement, François Pétis de La Croix a voulu donner un sens à sa " collecte » de contes orientaux. L'examen de la mise en recueil de ses sources diverses a ainsi révélé sous la proclamation liminaire d'un ordre moral, un questionnement plus large qui touche autant le sens du recueil: instruire, plaire, convaincre? que sa finalité : pourquoi raconter des histoires? Finalement, il me semble que le recueil met en question la réception du conte mais aussi le rapport entre l'intention du narrateur - ici, la nourrice et sa volonté " didactique »-, et celle de l'auteur, c'est-à-dire l'ordonnateur du recueil. Soit ces intentions coïncident et le conte se dote d'une fonction morale - ce sont les analyses de Marie-Louise Dufresnoy ou même de Roger Laufer -, soit elles divergent et on s'engage sur une nouvelle voie qui conduira le conte jusqu'aux architectures vertigineuses d'un Crébillon. La mise en recueil est une mise en question du conte.

\section{NOTES}

1. Pour un point sur cette question débattue, nous renvoyons à notre introduction de la nouvelle édition critique des Mille et Un Jours, «Bibliothèque des génies et des fées », Champion, à paraître courant 2004.

2. F. Pétis de La Croix, Les Mille et Un Jours, édition de Paul Sebag, C. Bourgois éditeur, 1981, p. 34. Toutes les références se feront dans cette édition.

3. Il en fait mention dans son Journal du sieur Pétis de La Croix renfermant tout ce qu'il a vu et fait en Orient, éd. M. Langlès, 1810.

4. On lit dans la préface du recueil : « [le dervis Moclès] confia son manuscrit au sieur Pétis de La Croix, qui était en liaison d'amitié avec lui à Ispahan en 1675, et même il lui permit d'en prendre une copie », Les Mille et Un Jours, p. 33.

5. Dans le conte original, Sayf-el-Muluk épouse Badi al Jamal, voir P. Sebag, Dossier, Les Mille et Un Jours, p. 502.

6. Pour plus de précisions ainsi que pour les références des différents manuscrits consultés par Pétis de La Croix, nous renvoyons au Dossier très complet de Paul Sebag à la fin de son édition du recueil, p. 495-508.

7. F. Pétis de La Croix, Histoire du Prince Calaf et de la princesse de la Chine, dossier par Paul Sebag, L'Harmattan, 2000, p. 116-119.

8. Paul Sebag interprète cette recomposition dans le sens de la vocation didactique du recueil : « les éléments empruntés à ces multiples sources ont été combinés, modifiés, abrégés ou développés et harmonieusement fondus pour composer un nouveau conte qui sert l'intention didactique de la conteuse. On ne peut comparer le texte publié par F. Pétis de La Croix avec les divers textes qu'il a mis à contribution sans admirer le talent avec lequel il a réussi à composer un récit d'une parfaite cohérence ", Notice du 
conte d'Aboulcassem Basri, Les Mille et Un Jours, p. 496. On pourrait également se demander si le conteur ne témoigne pas ainsi d'un certain art du roman.

9. Les Mille et Un Jours, p. 107.

10. Ibid., p. 78.

11. On peut peut-être lire ici un reproche implicite de l'auteur du recueil à son prédécesseur Antoine Galland qui a attendu le tome 3 des Mille et Une Nuits pour supprimer le « refrain » de Dinazarde : «comme cette répétition a choqué plusieurs personnes d'esprit, on l'a retranchée pour s'accommoder à leur délicatesse ", Les Mille et Une Nuits, Garnier Flammarion, 1965, t. 1, p. 226.

12. Dans une thèse intitulée « Lesage et Les Mille et Un Jours de François Pétis de La Croix », Christophe Balaÿ donne des précisions sur le remaniement du manuscrit turc : le conte original est focalisé sur les aventures du prince et de son confident Sy-Fuz (Symorgue chez Pétis de La Croix) et le rôle de Farrukhnaz est très réduit, Nanterre, 1979, voir p. 154-155.

13. L'expression revient plusieurs fois : il faut « découvrir son cœur », ne rien « cacher » à son roi.

14. Ainsi Malek, le tisserand toujours gai : « malgré mes ris et mes chants, je suis peutêtre le plus malheureux des hommes ", Les Mille et Un Jours, p. 268.

15. Dans l'édition de Paul Sebag, 193 pages contre 232 pages.

16. Roger Laufer analyse Les Mille et Un Jours comme une œuvre à part entière de Lesage.

17. Roger Laufer, Lesage ou le métier de romancier, Gallimard, 1971, p. 230-231.

18. Il propose une analyse structuraliste des relations amoureuses des personnages de différents contes, ibid., p. 236-238.

19. Il est vrai que le prince de Perse paraît bien passif face aux tribulations de Symorgue. On apprend cependant qu'il n'a pu se résoudre à attendre le retour de son confident puisqu'il est fait prisonnier par la magicienne Mehrefza, Les Mille et Un Jours, p. 479.

20. Cette rencontre a lieu cependant au prix d'une analepse : Farrukhnaz ne raconte pas son songe heureux au début du recueil mais à la fin, Les Mille et Un Jours, p. 469. 21. Selon Christophe Balaÿ, les contes originaux sont des contes édifiants destinés aux princes, des « miroirs des princes », thèse citée, p. 186. Nous n'avons pu cependant vérifier cette information.

22. Marie-Louise Dufresnoy, L'Idée de progrès et la Diffusion de la matière d'Orient, CDU, 1960 , p. 57-59.

23. « Histoire d'Hormoz surnommé le roi sans chagrin », Les Mille et Un Jours, p. 297.

24. " Histoire de Calaf et de la princesse de la Chine », ibid., p. 176.

25. Pour une analyse plus détaillée de cet aspect et du rôle de la parodie dans le recueil voir, C. Bahier-Porte, « Le laboratoire des Mille et Un Jours de Pétis de La Croix et Lesage ", dans R. Jomand-Baudry, J.-F. Perrin, Le Conte merveilleux au XVIII ${ }^{e}$ siècle - Une poétique expérimentale, Kimé, 2002, p. 23-41.

26. C'est également un des principes essentiels du roman picaresque à partir $d u \mathrm{XVI}^{\mathrm{e}}$ siècle, sauf que ce dernier, à l'origine en tout cas, consacre l'affliction tandis que les contes persans choisissent une certaine forme de joie.

27. Il s'agit des « charmes » des princesses négresses.

28. Pour Zélica dans l'histoire d'Atalmuk, il est possible de soupçonner une disparition volontaire, voir C. Bahier-Porte, art. cité, p. 38.

29. Sur cet aspect, voir Nadine Jasmin, Naissance du conte féminin - Mots et Merveilles, les contes de fées de Madame d'Aulnoy, Champion, 2002, p. 125-194. 


\section{RÉSUMÉS}

Programme d'ordre didactique : la narratrice, nourrice d'une jeune princesse, prétend prouver qu'il y a des hommes fidèles. Or, si l'on regarde de plus près la structure du recueil d'un point de vue narratologique puis thématique, on se rend compte d'une part que le récit-cadre principal accueille un second récit-cadre, d'autre part que ce recueil dans le recueil met en question l'optimisme démonstratif de la nourrice. La mise en recueil des contes des Mille et Un Jours révèle alors une double interrogation : sur l'insatiable quête du bonheur inhérente à la nature humaine mais aussi sur le rapport entre le conte et la satisfaction, de « l'auteur » comme du lecteur.

Collecting one thousand and one days. In order to compose One Thousand and One Days, Collection of Persian Tales, François Pétis de La Croix collected a number of tales of various origins which he organised according to a didactic programme: the female narrator, the wet nurse of a young princess, pretends she wants to prove some men are faithful. However, a closer look at the collection from a narratological and then thematic perspective reveals that the main frame includes another frame that puts the nurse's demonstrative optimism into question. The act of collecting these tales reveals a double questioning on the insatiable quest for happiness inherent to human nature, and on the connections between fairy tales and gratification, of the " author " as well as of the reader.

\section{AUTEUR}

\section{CHRISTELLE BAHIER-PORTE}

Université Lyon 3 - UMR LIRE 